\title{
Therapy efficacy of hyperreative rhinitis by the combination of intranasal steroid and oral H1-antihistamine
}

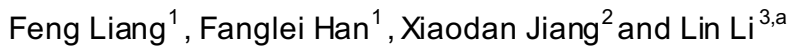 \\ ${ }^{1}$ Department of Anesthesiology, China- Japan Union Hospital, Jilin University, 130033, China \\ ${ }^{2}$ Otorhinolary, Affiliated Hostipal og Qingdao University, Qingdao,266555, China \\ ${ }^{3}$ Dept. Otolaryngolgy and head \& Neck, China- Japan Union Hospital, Jilin University, 130033, China
}

\begin{abstract}
Background Hyperreative rhinitis (HRR) is identified as rhinitis which has at least two symptoms such as sneeze, itches, watery secretion and nasal blockage. Little is known about the pathology of HRR, as well as its clinical features and treatment. Objective To investigate efficacy HRR by combined therapy with intranasal steroids and antihistamines Methods The age, gender, predisposing factors and clinical symptoms of $200 \mathrm{HRR}$ patients were analyzed. Both intranasal steroids and antihistamines were applied for 8 weeks. Symptoms were assessed with VAS score before and after the treatment. Result: Before the therapy, $87 \%$ of HRR patients showed persistent symptoms; $69 \%$ complained about severe and persistent effects on the quality on the life; sneezing (96\%) and rhinorrhea (78\%) were the top 2 symptoms reported; All patients completed the 8-wk combined therapy with intranasal steroids and antihistamines; $96 \%$ patients were satisfied with the therapy, with all symptoms except wheezing have been clear relief $(p<0.05)$; the remaining $4 \%$ reported the unsatisfied result of the therapy. Conclusion The combined therapy with intranasal steroids and antihistamines controlled most of clinical symptoms of HRR.
\end{abstract}

\section{Instruction}

Rhinitis is an extremely common rhinopathy. Rhinit is has been divided into four main categories: infectious rhinitis, allergic rhinitis (AR), structural rhinitis, and non-allerg ic rhinitis (NAR). Differential diagnosis of AR from NAR is often difficult because of their similar clinical presentation. NAR is now a diagnosis of exclusion characterized by similar symptoms to A R, but with no allergic sensitization-related symptoms and signs of infection [1]. Hyperreactive rhin itis (HRR) is identified as the combination of AR and NAR because of similar sympto ms.

HRR affects a significant number of patients in clinical practice. Studies have shown an increase in the prevalence of asthma in patients with HRR[2] it has been hypothesized that there was a link between upper and lower airways beyond allergy associated inflammation. Our current study focused on the efficacy of one co mbined therapy with intranasal steroid and oral antihistamine on HRR.

\section{Materials and methods}

\footnotetext{
a Corresponding author: 1ilin01@jlu.edu.cn
} 


\subsection{Clinical data}

200 consecutive untreated patients (110 females and 90 males), who were diagnosed of HRR, participated the study. The cases were collected from the files recorede between January, 2015 and December, 2015, from the Department of Otolaryngology, Head and Neck Surgery of the University of China-Japan Union Hospital attached to Jilin University, China. The study was approved by the institutional review board of China-Japan Union Hospital. All the participants provided written informed consent before the study.

\subsection{Clinical questionnaire and medical interview}

Data of the initial evaluation of NAR patients were extracted from the med ical history obtained during their first visit to our center. A clinical questionnaire, a complete file of physical examination and symptoms were included. Visual Analogue Scale (VAS) was used to evaluate the severity of nasal symptoms before and after the therapy. The range of the score was 0-3: higher scores indicated more severe symptoms, and score 0 indicated no symptoms. One year follow-up evaluation was also conducted after the treatment. NAR was defined by the presence of two or more nasal symptoms (sneezing, itching, rhinorrhea, or nasal obstruction) plus a negative SPT response and serum IgE level specific to aeroallergens.

\subsection{Therapy procedures}

Patients were treated with once-daily intranasal budesonide for 8 weeks. $200 \mathrm{mg}$ Budesonide were given to patients aged $<11$ years at study entry and $400 \mathrm{mg}$ to patients aged $>11$ years at study entry. Simu ltaneously, Loratadine was administered orally to patients ( $>12$ years old) with $10 \mathrm{mg}$ per day before sleeping for 8 weeks

\subsection{Statistical analysis}

Descriptive statistics (frequency, mean, median, SD, and range) were used to analyzed the clinical features of HRR. Paired t-test was used to analyze the therapy results. The data were analyzed with SPSS for Windows 17.0 (SPSS, Inc, Chicago, Ill). P-values $<0.05$ were considered statistical significant.

\section{Result}

\subsection{Age and sex distribution}

A total of 200 patients were survey in our study. They aged from 4 to 76 , with an average of $38 \pm 14$ ) years old $87 \%$ of patients showed persistent symptoms and $69 \%$ complained about severe and persistent effects on the quality on the life; there was no significant difference in the age between male and female patients $(\mathrm{p}=0.983)$

\subsection{The efficacy of an 8-wk combined therapy of intranasal steroid and oral antihistamine}

All 200 patients were contacted by telephone for follow-up evaluation after 8-wk therapy. All the symptoms were significantly decreased after the 8 -wk therapy $(\mathrm{p}<0.05) 96 \%$ patients were satisfied 
with the therapy, with all symptoms have been clear relief $(p<0.05)$; the remaining $4 \%$ reported the unsatisfied result of the therapy but still has decreased VAS after the combined treatment. (Table 1).

Table 1. All the HRR symptoms were significantly decreased after the 8-wk combined therapy

\begin{tabular}{lllllllllll}
\hline & \multicolumn{3}{c}{ before the treatment } & \multicolumn{3}{c}{ after the treatment } & z & $\mathrm{p}$ \\
VAS & 0 & 1 & 2 & 3 & 0 & 1 & 2 & 3 & & \\
\hline sneezing(case) & 8 & 24 & 64 & 42 & 80 & 52 & 20 & 0 & -5.12 & 0 \\
secretion(case) & 16 & 20 & 36 & 80 & 104 & 44 & 4 & 0 & -5.18 & 0 \\
blockage(case) & 20 & 32 & 42 & 44 & 128 & 20 & 4 & 0 & -5.07 & 0 \\
ithcy(case) & 44 & 36 & 24 & 48 & 124 & 28 & 0 & 0 & -4.46 & 0 \\
\hline
\end{tabular}

\section{Discussion}

Both NAR and AR are associated with the nasal mucosa hyper-responsiveness and resulted in similar symptoms and physical signs, so we identified the hyperreactive rhinitis (HRR) according to symptoms. Currently, the diagnos is of HRR relies on the clinical sympto ms.

Standard treatment reference of HRR has not been established yet. Similar treatment designed for AR has been used to treat to HRR due to the shared symptoms and morbidity between this two types of rhinitis. Studies showed that intranasal steroids such as Budesonide, Fluticasone and Mometasone, could relieve the edema condition of nasal cavity and decrease the symptoms of NAR. Rinne [3] reported that intranasal steroids could success fully control the recurrence of the NAR. Patients with sneezing as a predominant symptom responded well to oral antih istamines. The combination of Flunisolide and Loratadine showed better clinical efficacy than intranasal steroid alone [4]. In our study, $96 \%$ patients showed alleviated symptoms of nose, eye and lower airway after a combined therapy of intranasal steroids and oral anti-histamines for 8 weeks, indicating the therapy was effective in HRR treatment. The histamine receptor 3 was the decisive factors in nasal neural system, whereas the target of Loratadine is histamine receptor 1 that main ly responsible to the allergic reaction. Studies have showed that Azelastine decreased all the symptoms of NAR [5] and AR. Azelastine is the intranasal antihistamine commercially available. Studies showed that Azelstine interfered with vasoactive neuroperptide substance $\mathrm{P}$ and inhibited the production of leukotrienes $\mathrm{B} 4$ and $\mathrm{C}$, which might possibly decrease the expression of nuclear factor- $\mathrm{kB}$, which was a transcription factor for multiple proinflammatory substances[6]. The efficacy of intranasal Azelastine might be further explored to replace Loratadine in the combination therapy to treat NAR and AR.

In conclusion, HRR had it own clinical feature, such as high risk population is adults (30-39), slightly more in female, The comb ined therapy of intranasal steroid and oral histamines was effective in controlling HRR symptoms. In addition, the individualized therapy procedure needs further consideration for the inter-individual variability of the therapy efficacy observed in our study.

\section{Acknowledgement}

All authors declared no conflict of interest in the research. The work presented in this report is the subject of a pending patent filed by the China-Japan Union Hospital of Jilin University (201510382781.X). This study was supported by the National Natural Science Foundation of China (811007020), the Health and Family Planning Foundation of Jilin Province (20152046), the Science and Technology Development Plan Foundation of Jilin Province (20160101070JC), and the Norman Bethune Program of Jilin University (2015305). 


\section{References}

1. Bousquet J, Fokkens W, Burney P, et al.. Allergy. 63 842-853, (2008)

2. Chawes BL, Bønnelykke K, Kreiner-Møller E, et al. J Allergy Clin Immunol. 2010; 126 (3) 567-573.e 1-8(2010)

3. Rinne J, Simola M, Malmberg H, et al. J Allergy Clin Immunol. 109(3) 26-432 (2002)

4. Purello-D'A mbrosio F, Isola S, Ricciardi L, et al. Clin Exp Allergy.29(8) 143-1147 (199 9)

5. Banov $\mathrm{CH}$, Lieberman P. Vasomotor Rhinit is Study Groups. Ann Allergy Asthma Immun ol.86 28-35 (2001)

6. Greiner AN, Meltzer EO. J Allergy Clin Immunol. 118(5)985-996 ( 2006) 